\title{
Covid-19 Case Statistics in Europe Show Moderate Negative Correlation with Vaccination Level
}

\author{
Serge Dolgikh ${ }^{10000-0001-5929-8954] ~}$ \\ Dept. of Information Technology, \\ National Aviation University, Kyiv
}

\begin{abstract}
Based on the statistics of Covid-19 cases and vaccination level in the last quarter of 2021, a moderate negative correlation was detected between the level of vaccination and current and peak case numbers in a number of European health jurisdictions. Statistically significant correlation was detected after taking into account differences in the local phase of the cases curve, by comparing statistics at the similar phase such as near the top of a local wave rather than current. These results may indicate a certain level of effectiveness of vaccines in preventing Covid-19 spread in the population, though analysis of other factors is still needed for a confident conclusion.
\end{abstract}

Keywords: Epidemiology, Covid-19, BCG, vaccination

\section{Introduction}

We examined correlation between the vaccination level in a number of reporting European jurisdictions (parameter vacc) and Covid-19 case dynamics, measured by two parameters: the current Covid-19 cases (case-curr) and the peak Covid-19 cases (case-peak). The peak value was used due to the fact that epidemiological curves in different jurisdictions may not be aligned in time, and comparing current statistics may not produce correct and informative results.

The data for the analysis was obtained from open to the public sources.

\section{Data}

Vaccination and case count statistics were obtained from publicly available sources [1-3].

For the peak value, maximum Covid-19 case counts were taken in the period after May, 2021 when vaccination level in the reporting jurisdictions can be expected to have achieved a certain minimal threshold.

Case factors, current and peak, were measured as the number of reported cases, 7day average, per 1 million of population.

Vaccination level per jurisdiction was reported as the number of administered doses per capita of population. The number included administered single doses and cannot be considered as an exact measure of the fully vaccinated eligible population in the jurisdiction. 
For the correlation analysis, jurisdictions with populations above 1 million were selected to reduce population-related factor variation in the selected group.

The resulting set of statistics from 29 European national jurisdictions is presented in Table 1.

Table 1 National vaccination and case statistics, Europe (late fall, 2021)

\begin{tabular}{|l|r|r|r|r|}
\hline Country & $\begin{array}{c}\text { Population, } \\
\text { mln }\end{array}$ & $\begin{array}{c}\text { Vaccination } \\
\text { level, vacc }\end{array}$ & $\begin{array}{c}\text { Current cases, } \\
\text { case-curr }\end{array}$ & $\begin{array}{c}\text { Peak cases, } \\
\text { case-peak }\end{array}$ \\
\hline UK & 66.00 & 0.821 & 0.673 & 0.714 \\
\hline Portugal & 10.31 & 0.802 & 0.212 & 0.425 \\
\hline Ireland & 5.01 & 0.800 & 0.878 & 0.878 \\
\hline Spain & 47.35 & 0.799 & 0.120 & 0.543 \\
\hline Italy & 60.50 & 0.775 & 0.158 & 0.163 \\
\hline Denmark & 5.60 & 0.768 & 0.663 & 0.691 \\
\hline France & 67.00 & 0.757 & 0.276 & 0.355 \\
\hline Sweden & 10.10 & 0.751 & 0.089 & 0.117 \\
\hline Finland & 5.50 & 0.745 & 0.185 & 0.189 \\
\hline Norway & 5.40 & 0.733 & 0.380 & 0.380 \\
\hline Belgium & 11.40 & 0.733 & 1.388 & 1.388 \\
\hline Austria & 8.92 & 0.716 & 1.570 & 1.570 \\
\hline Netherlands & 17.20 & 0.710 & 1.254 & 1.277 \\
\hline Cyprus & 1.21 & 0.701 & 0.347 & 0.843 \\
\hline Germany & 82.80 & 0.701 & 0.617 & 0.641 \\
\hline Hungary & 9.75 & 0.687 & 0.951 & 0.968 \\
\hline Lithuania & 2.80 & 0.665 & 0.625 & 1.050 \\
\hline Switzerland & 8.64 & 0.663 & 0.612 & 0.647 \\
\hline Greece & 10.50 & 0.657 & 0.645 & 0.645 \\
\hline Czech Rep. & 10.70 & 0.608 & 1.629 & 1.629 \\
\hline Latvia & 1.90 & 0.557 & 0.432 & 1.316 \\
\hline Slovenia & 2.10 & 0.568 & 1.505 & 1.590 \\
\hline Serbia & 6.91 & 0.558 & 0.438 & 1.027 \\
\hline Estonia & 1.33 & 0.555 & 0.556 & 1.361 \\
\hline Poland & 38.00 & 0.537 & 0.540 & 0.553 \\
\hline Croatia & 4.10 & 0.489 & 1.110 & 1.349 \\
\hline Slovakia & 5.50 & 0.450 & 1.707 & 1.855 \\
\hline Romania & 19.30 & 0.376 & 0.147 & 0.778 \\
\hline Bulgaria & 6.93 & 0.231 & 0.368 & 0.690 \\
\hline & & & & \\
\hline
\end{tabular}

\section{Sources:}

European vaccination statistics data [1,2]

Covid-19 case statistics [2-4] and other sources [5,6] 


\section{Results}

\subsection{Negative Correlation}

Pearson's correlation coefficient was calculated for the ranges (vacc, case-curr) and (vacc, case-peak):

$$
K_{\text {curr }}=-0.135 ; K_{\text {peak }}=-0.382
$$

A negative value of the coefficient means that higher level of vaccination is correlated with lower case statistics in both cases.

As can be observed in (1), current case statistics show only a weak correlation with the level of vaccination. The cause becomes apparent after examination of case dynamics in specific jurisdictions. It can be observed that the time point of the analysis at which the values of the current cases range case-curr are recorded, can fall in different phases of the local case curves in different jurisdictions, as illustrated in Figure 1 ; instances with cases near the peak; on the descending arch and near the bottom of the local curve at the time point at which case statistics were recorded were observed.

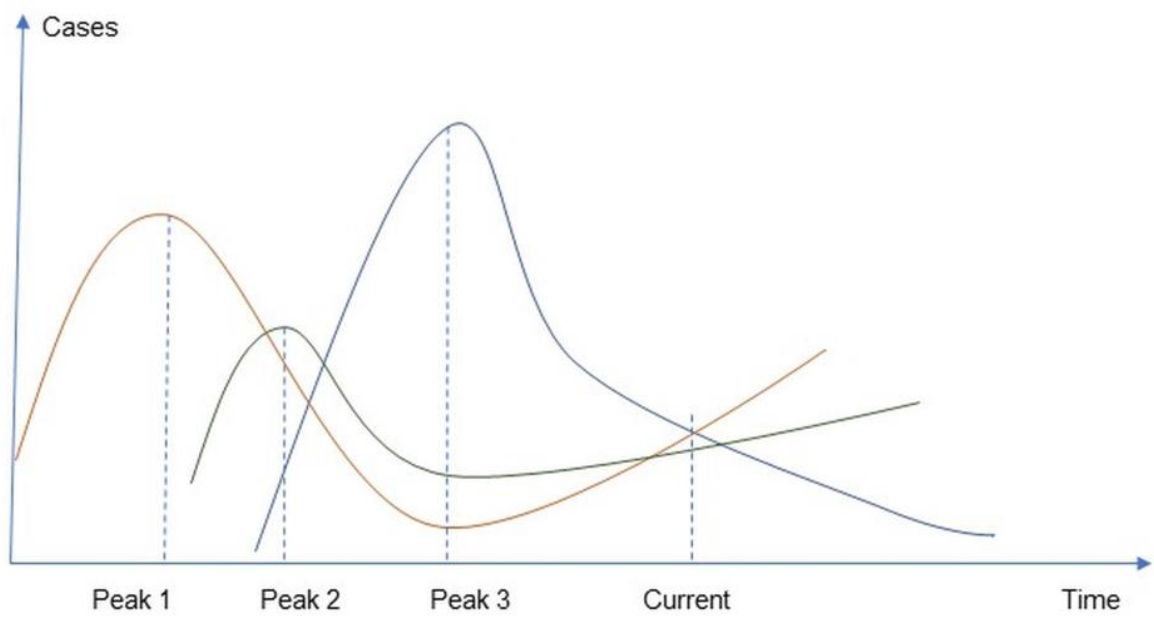

Fig.1 Case dynamics curves, current and peak values

For this reason, it was concluded that the peak parameter case-peak, representing the peak number of cases recorded in the jurisdiction in the reviewed period once vaccinations become available and a certain minimal level of vaccination was achieved, should provide a more correct description of the case dynamics in the jurisdiction.

As can be seen in (1), using peak case statistics resulted in a higher absolute value of the correlation coefficient, $K_{\text {peak }}=0.382$, indicating a more pronounced correlation between vaccination level and the statistics of the cases, with a p-value of 0.0407 , indicating a statistically significant correlation at the confidence level $\alpha=0.05$. 


\section{Conclusions}

A correlation analysis of the statistics of vaccination levels and Covid-19 case dynamics in European health jurisdictions showed statistically significant negative correlation between the level of vaccination and Covid-19 cases represented by local peaks of the case curve. While direct causation cannot be concluded from these results without an additional analysis of other essential factors of influence, it provides grounds for an expectation that higher vaccination levels can be associated with lower incidence of reported cases of the infection.

It needs to be emphasized that availability of open national statistics on key epidemiological parameters, including case incidence, immunization levels, hospitalizations and others contributed significantly to facilitate research and reduce the time and effort of the analysis in this work.

In conclusion it can be mentioned that a coordinated effective strategy including targeted immunization, correct, current and clear information, effective monitoring of the current epidemiological situation and environmental approaches to controlling the spread of the infection in the higher risk environments $[7,8]$ can be used to suppress the contagion more effectively while allowing a normal functioning of the society, to the extent possible.

\section{References}

1. Europe: Covid-19 vaccination rates. Statista, https://www.statista.com/statistics/1196071/covid-19-vaccination-rate-in-europe-bycountry/ (accessed 23.11.2021).

2. Coronavirus data Google https://www.google.com/covid19-map/ (23.11.2021).

3. Johns Hopkins University Medicine Coronavirus Resource Center https://coronavirus.jhu.edu/map.html (21.11.2021).

4. Worldometers: Population data, https://www.worldometers.info/world-population/ (21.11.2021).

5. ECDC Covid-19 hospital and care data. https://www.ecdc.europa.eu/en/publicationsdata/download-data-hospital-and-icu-admission-rates-and-current-occupancy-covid-19 (21.11.2021).

6. Finnish Institute of Health and Welfare: Covid-19 situation update, https://thl.fi/en/web/infectious-diseases-and-vaccinations/what-s-new/coronavirus-covid19-latest-updates/situation-update-on-coronavirus (15.11.2021).

7. Tabish M., Khatoon A., Alkahtani S. et al.: Approaches for prevention and environmental management of novel COVID-19. Environmental Science and Pollution Research, 28, 40311-40321 (2020).

8. Dolgikh, S.: Smart-Covid: intelligent solutions for higher risk environments, HAL archives-ouvertes, hal-02915459, version 2 (2020). 